\title{
ON SUBMAXIMAL SPACES
}

\author{
JULIAN DONTCHEV
}

\begin{abstract}
A topological space $X$ is called submaximal if every dense subset of $X$ is open. In this paper, which is an enlarged version of Section 3 in [7], we characterize submaximal spaces using various topological notions. We study the connections between submaximal and related spaces as well as we improve some results concerning submaximal space achieved by Mahmoud and Rose in [7].
\end{abstract}

\section{Introduction}

Submaximal spaces were defined by Bourbaki [1] and hence a lot of results in general topology involved the defined notion. In various papers, for example [3], submaximal spaces were considered significantly. In a recent paper [7] a separate section investigated submaximality. Interesting and important results were proved using the notions of generalized open sets: semi-open, preopen, $\alpha$-open, etc. The aim of this paper is to study more deeply the notion of submaximality and to improve some of the results given by Mahmound and Rose in [7].

Throughout this paper we consider topological spaces on which no separation axioms are assumed unless explicitly stated. The topology of a space is denoted by $\tau$ and $(X, \tau)$ will be replaced by $X$ if there is no chance for confusion. The word "iff" means "if and only if", a "space" will always mean a topological space and the symbol $\square$ is used to indicate the end or omission of a proof. For $A \subset X$, the closure, the interior and the boundary of $A$ in $X$ are denoted by $\mathrm{Cl} A, \operatorname{Int} A$ and $\operatorname{Fr} A$, respectively. In places where there is no chance for confusion $\bar{A}$ stands for $\mathrm{Cl} A$.

The following definition is a collection of all notions of different classes of sets used in this paper.

Definition 1. A subset $A$ of a space $(X, \tau)$ is called:

(1) a regular open set if $A=\operatorname{Int} \bar{A}$,

Received February 18, 1994; revised October 29, 1994.

1991 Mathematics Subject Classification. Primary 54F65, 54G05; Secondary 54H05, 54G99.

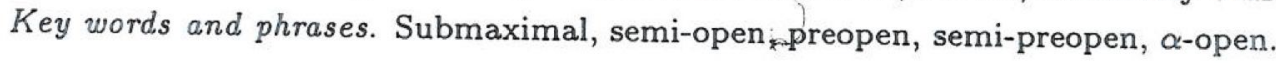


(2) a preopen set [8] if $A \subset \operatorname{Int} \bar{A}$,

(3) a semi-open set [5] if $A \subset \overline{\operatorname{Int} A}$,

(4) a $\alpha$-open set [11] if $A \subset \operatorname{Int} \overline{\operatorname{Int} A \text {, }}$

(5) a locally closed set [1] if $A=U \cap F$, where $U$ is open and $F$ is closed,

(6) a $B$-set [13] if $A=U \cap F$, where $U$ is open and $F$ is semi-closed,

(7) a generalized closed set (g-closed set) [4] if $\bar{A} \subset U$ whenever $A \subset U$ and $U$ is open,

(8) a residual set if $\operatorname{Int} A=\varnothing$.

We denote the collection of all regular open (resp. preopen, semi-open) sets in $(X, \tau)$ by $\mathrm{RO}(X)$ (resp. $\mathrm{PO}(X), \mathrm{SO}(X)$ ). The family of all dense subsets of $(X, \tau)$ will be denoted by $\mathcal{D}(X)$ and the family of their interiors by $\mathcal{D}^{0}(X)$.

Definition 2. A topological space $(X, \tau)$ is called:

(1) a door-space if each subset of $X$ is either open or closed,

(2) S-space (or has the S-topology) [6] if every subset which contains a non-void open subset is open,

(3) an irreducible space [2] if every two non-void open subset of $X$ intersect,

(4) d-compact [3] if every cover of $X$ by dense subsets has a finite subcover.

Definition 3. A space $X$ is called submaximal [1] iff every dense subset of $X$ is open.

In [7] it is proved in Theorem 5 that semi-open subsets of submaximal spaces are submaximal. We close this first introductory section with a result which improves the result in [7] and which will be used often throughout the paper.

Theorem 1.1. Every subspace $S$ of a submaximal space $X$ is submaximal.

Proof. Let $A$ be a dense subset of $S$. Then $\bar{A} \cap S=S$ and so $S \subset \bar{A}$. Since $A \cup(X \backslash \bar{A})$ is dense in $X$, then it is an open subset of $X$. Hence $S \cap(A \cup(X \backslash \bar{A}))=A$ is open in $S$ or equivalently $S$ is submaximal.

Remark 1.2. This paper is based on [10].

\section{The simple extension of topology over a set}

Given a space $(X, \tau)$ and a subset $A \subset X$, we denote by $\tau(A)$ the simple extension of $\tau$ over $A$. [14], i.e., the collection of sets $U \cup(V \cap A)$, where $U \in \tau$ and $V \in \tau$. Note that $\tau(A)$ is a topology on $X$ finer than $\tau$. The next theorems study some connections between dense sets and the simple extension of a given topology over a set, which will help us in establishing some new results about submaximal spaces. 
Theorem 2.1. If $A$ is a dense subset of the space $(X, \tau)$, then $A$ is also dense in $(X, \tau(A))$.

Theorem 2.2. Let $A$ be a dense subset of the space $(X, \tau)$. Then for every open subset $G$ of the space $(X, \tau(A))$ we have $\mathrm{Cl}_{\tau} G=\mathrm{Cl}_{\tau(A)} G$ and for every closed subset $S$ of the space $(X, \tau(A))$ we have $\operatorname{Int}_{\tau} S=\operatorname{Int}_{\tau(A)} S$.

Proof. Let first $U \in \tau$. Since $\tau \subset \tau(A)$, then $\mathrm{Cl}_{\tau(A)} U \subset \mathrm{Cl}_{\tau} U$. For the reverse inclusion let $x \in \mathrm{Cl}_{\tau} U$ and let $G$ be an open neighborhood of $x$ in $(X, \tau(A))$. Then $x \in G=H \cup(K \cap A)$, where $H, K \in \tau$. If $x \in H$, then $H \cap U \neq \varnothing$ and thus $G \cap U \neq \varnothing$. Again if $x \in K \cap A \subset K$, then $K \cap U \neq \varnothing$ and hence $K \cap U \cap A \neq \varnothing$, since $K \cap U \in \tau$ and since $A$ is dense. Thus also in this case $G \cap U \neq \varnothing$ and hence $x \in \mathrm{Cl}_{\tau(A)} U$. We have shown that $\mathrm{Cl}_{\tau} U=\mathrm{Cl}_{\tau(A)} U$ for each $U \in \tau$.

Let next $G \in \tau(A)$. Then $G=H \cup(K \cap A)$, where $H, K \in \tau$. From the above proved it is clear that $\mathrm{Cl}_{\tau} H=\mathrm{Cl}_{\tau(A)} H$. Since $K \in \tau(A)$ and since $A$ is dense in $(X, \tau(A))$, then according to Theorem $2.1 \mathrm{Cl}_{\tau(A)}(K \cap A)=\mathrm{Cl}_{\tau(A)} K=\mathrm{Cl}_{\tau} K=\mathrm{Cl}_{\tau}(K \cap A)$. Thus

$$
\mathrm{Cl}_{\tau(A)} G=\mathrm{Cl}_{\tau} H \cup \mathrm{Cl}_{\tau}(K \cap A)=\mathrm{Cl}_{\tau}(H \cup(K \cap A))=\mathrm{Cl}_{\tau} G \text {. }
$$

From this it follows that if $S$ is a closed subset of $(X, \tau(A))$, then $\mathrm{Cl}_{\tau(A)}(X \backslash S)=$ $\mathrm{Cl}_{\tau}(X \backslash S)$ or equivalently $X \backslash \operatorname{Int}_{\tau(A)} S=X \backslash \operatorname{Int}_{\tau} S$ or equivalently $\operatorname{Int}_{\tau(A)} S=\operatorname{Int}_{\tau} S$.

Corollary 2.3. If $A$ is a dense subset of the space $(X, \tau)$, then for every $U \in \tau(A)$ we have $\operatorname{Int}_{\tau} \mathrm{Cl}_{\tau} U=\operatorname{Int}_{\tau(A)} \mathrm{Cl}_{\tau(A)} U$. Hence the set $U$ is a regular open subset of $(X, \tau)$ iff it is regular open in $(X, \tau(A))$.

Proof. According to Theorem $2.2 \operatorname{Int}_{\tau} \mathrm{Cl}_{\tau} U=\operatorname{Int}_{\tau} \mathrm{Cl}_{\tau(A)} U=\operatorname{Int}_{\tau(A)} \mathrm{Cl}_{\tau(A)} U$.

Corollary 2.4. If $A$ is a dense subset of the connected space $X$, then $(X, \tau(A))$ is also connected.

Proof. Due to the corollary above, when $A$ is dense in $(X, \tau)$, the regular closed sets of $(X, \tau(A))$ are precisely those of $(X, \tau)$ and thus clopen sets are the same for both spaces.

\section{The characterization of submaximal spaces}

Theorem 3.1. For a space $(X, \tau)$ the following conditions are equivalent:

(1) $X$ is submaximal.

(2) If $\tau(A)$ is a simple extension of topology over $A$ on $X$ finer than $\tau$, such that $\mathrm{RO}(X, \tau)=\mathrm{RO}(X, \tau(A))$, then $\tau=\tau(A)$.

(3) If $\tau(A)$ is a simple extension of topology over $A$ on $X$ finer than $\tau$, such that each regular open subset of $(X, \tau(A))$ is open in $(X, \tau)$, then $\tau=\tau(A)$. 
Proof. (1) $\Rightarrow(3)$ Let $U \in \tau(A)$. Since $\operatorname{Int}_{\tau(A)} \mathrm{Cl}_{\tau(A)} U=V$ is regular open in $(X, \tau(A))$, then it is open in $(X, \tau)$. Since $U \subset \operatorname{Int}_{\tau(A)} \mathrm{Cl}_{\tau(A)} U=V \subset \mathrm{Cl}_{\tau(A)} U \subset \mathrm{Cl}_{\tau} U$, then $U$ is dense in $V$. Since $V$ is submaximal according to Theorem 1.1, then $U$ is open in $V$. By transitivity $U \in \tau$. Hence $\tau=\tau(A)$.

(3) $\Rightarrow(2)$ is trivial.

$(2) \Rightarrow(1)$ Let $A$ be dense in $(X, \tau)$. Since $\tau \subset \tau(A)$ and since the regular open subsets of $(X, \tau(A))$ and $(X, \tau)$ coincide according to Corollary 2.3 , then by assumption $\tau=\tau(A)$. Hence $A \in \tau$ and thus $(X, \tau)$ is submaximal.

Remark 3.2. A more general equivalence of (2) and (1) in the theorem above is well-known. For any topology $\tau$ on a set $X$, the semiregularization topology, $\tau_{s} \subset \tau$, has for a base the regular open sets in $(X, \tau)$. Let $\tau$ be a topology on $X$, and let $\mathcal{S}=\{\sigma: \sigma$ is a topology on $X$ and $\left.\sigma_{s}=\tau_{s}\right\}$. Then $(X, \tau)$ is submaximal iff $\tau$ is maximal in $\mathcal{S}$ [12].

Theorem 3.3. For a space $(X, \tau)$ the following conditions are equivalent:

(1) $X$ is submaximal.

(4) For any $A \subset X$, the subspace $\operatorname{Fr} A=\bar{A} \backslash \operatorname{Int} A=\bar{A} \cap \overline{X \backslash A}$ is discrete.

Proof. (1) $\Rightarrow(4)$ Let $x \in \operatorname{Fr} A$. Since $A$ is dense in $\bar{A}$, then so is $A \cup\{x\}$. Since $\bar{A}$ is submaximal according to Theorem 1.1, then $A \cup\{x\}=\bar{A} \cap U$, where $U$ is open in $X$. In the same way it can be seen that $(X \backslash A) \cup\{x\}=\overline{X \backslash A} \cap V$, where $V$ is open in $X$. Thus $\{x\}=(A \cup\{x\}) \cap((X \backslash A) \cup\{x\})=\bar{A} \cap \overline{X \backslash A} \cap U \cap V$. Hence $\{x\}$ is open in $\operatorname{Fr} A$ and so $\operatorname{Fr} A$ is discrete.

(4) $\Rightarrow$ (1) Let $A$ be dense in $X$. By (4) $\bar{A} \backslash \operatorname{Int} A=X \backslash \operatorname{Int} A$ is discrete and thus $A \backslash \operatorname{Int} A$ is its open subset. Hence $A \backslash \operatorname{Int} A=(X \backslash \operatorname{Int} A) \cup U$, where $U$ is open in $X$. Thus $A \backslash \operatorname{Int} A \subset U$ and so $A \backslash \operatorname{Int} A \subset U \backslash \operatorname{Int} A$. For the reverse inclusion if $x \in U \backslash \operatorname{Int} A$, then $x \in(X \backslash \operatorname{Int} A) \cap U=A \backslash \operatorname{Int} A$. This shows that $A \backslash \operatorname{Int} A=U \backslash \operatorname{Int} A$ and hence $A=U \cup \operatorname{Int} A$. Thus $A$ is open in $X$.

In [7] Mahmoud and Rose show in Lemma 5 that if $(X, \tau)$ is submaximal space, then $\mathrm{PO}(X, \tau)=\tau$. In what follows we give an alternative proof of this result showing that it is the necessary and sufficient condition for a space in order to be submaximal.

Theorem 3.4. For a space $X$ the following are equivalent:

(1) $X$ is submaximal.

(5) Every preopen subset of $X$ is open.

Proof. (1) $\Rightarrow(5)$ Let $A$ be preopen in $X$. Then $A \subset \operatorname{Int} \bar{A}$. Since $A$ is dense in $\bar{A}$ and since $\bar{A}$ is submaximal according to Theorem 1.1, then $A$ is open in $\bar{A}$. Thus $A$ is open also in $\operatorname{Int} \bar{A}$. Since $\operatorname{Int} \bar{A}$ is open in $X$, then also $A$ is open in $X$.

(5) $\Rightarrow(1)$ Let $A$ be dense in $X$. Since $A \subset X=\operatorname{Int} X=\operatorname{Int} \bar{A}$, then $A$ is preopen and by (5) open. This shows that $X$ is submaximal. 
Corollary 3.5. In submaximal spaces compactness coincides with strong compactness (that is every cover of preopen sets has a finite subcover [9]).

Theorem 3.6. For a space $X$ the following are equivalent:

(1) $X$ is submaximal.

(6) The boundary $\operatorname{Fr} A$ of each subset $A \subset X$ has no accumulation points.

(7) Every residual set is without accumulation points.

Proof. (4) $\Rightarrow(6) \operatorname{By}(4) \operatorname{Fr} A$ is discrete. Thus it has no accumulation points, since it is closed.

(6) $\Rightarrow$ (7) If $\operatorname{Int} A=\varnothing$, then $A \subset \bar{A}=\bar{A} \backslash \operatorname{Int} A=\operatorname{Fr} A$ and by (6) $A$ has no accumulation points.

$(7) \Rightarrow(1)$ By (7) every residual set is closed. Thus every dense subset is open.

\section{Locally closed sets and $T_{\frac{1}{2}}$-spaces}

Recall that a space $X$ is called a $T_{\frac{1}{2}}$-space if it satisfies the following equivalent conditions:

(1) Every g-closed set is closed.

(2) Every singleton is either open or closed.

(3) The complement of a finite set is locally closed.

Theorem 4.1. Every subset $A$ of a submaximal space $X$ is locally closed and hence $X$ is a $T_{\frac{1}{2}}$-space.

Proof. Since by Theorem $1.1 \bar{A}$ is submaximal and since $A$ is its dense subset, then $A$ is open in $\bar{A}$. Hence $A=U \cap \bar{A}$, where $U$ is open and $\bar{A}$ is closed. Thus $\mathrm{A}$ is locally closed.

The more general result is as follows:

Theorem 4.2. For a space $X$ the following are equivalent:

(1) $X$ is submaximal.

(8) Every subset of $X$ is locally closed.

Proof. (1) $\Rightarrow$ (8) Theorem 4.1.

(8) $\Rightarrow$ (1) If $A$ is dense in $X$, then by (8) $A=U \cap C$, where $U$ is open and $C$ is closed. Since $A \subset C$, then $X=\bar{A} \subset C$. So $C=X$ and hence $A=U$. Thus $A$ is open in $X$.

Note that $T_{\frac{1}{2}}$-spaces need not be submaximal; not even metric spaces are always submaximal. For, the real line with the usual topology shows. However the next theorem gives the necessary condition over which $T_{\frac{1}{2}}$-spaces are submaximal.

Theorem 4.3. Every d-compact $T_{\frac{1}{2}}$-space $X$ is submaximal. 
Proof. Let $A$ be a residual subset of $X$ and let $x \in A$. If $\{x\}$ is open, then $x \in \operatorname{Int} A$ and this contradicts the assumption that $A$ is a residual set. Since $X$ is a $T_{\frac{1}{2}}$-space, then $\{x\}$ is closed for each $x \in A$. Since $X$ is d-compact, then $A$ is finite. Hence $A$ is closed being the finite union of closed singletons. Thus $X$ is submaximal.

Corollary 4.4. For a space $X$ the following are equivalent:

(1) $X$ is d-compact and submaximal.

(2) $X$ is a d-compact $T_{\frac{1}{2}}$-space.

Proof. Theorem 4.1 and Theorem 4.3.

Remark 4.5. Note that a d-compact space need not be submaximal. For, consider the space $X=\{a, b, c\}$ with the only non-trivial open set $\{a\}$.

On the other hand a submaximal space need not be d-compact. An example of such space is the real line where the non-void open sets are all sets that contain the zero-point.

\section{More on submaximal and related spaces}

Theorem 5.1. If $(X, \tau)$ is submaximal and $U \subset X$, then $U$ is open iff it is the intersection of a dense and a regular open set.

Proof. We need to show only that for every open set $U$ we have $U=D \cap V$, where $D$ is dense and $V$ is regular open, since the reverse inclusion is trivial. Clearly $U \subset \operatorname{Int} \bar{U}$. Thus $U=\bar{U} \backslash(\bar{U} \backslash U)=\bar{U} \cap(X \backslash(\bar{U} \backslash U))=\operatorname{Int} \bar{U} \cap(U \cup(X \backslash \bar{U}))$, where Int $\bar{U}=V$ is regular open and $U \cup(X \backslash \bar{U})=D$ is dense.

Theorem 5.2. The Alexandrov-compactification $X_{p}$ of every infinite discrete space is a submaximal space.

Proof. Let $A$ be a dense subset of $X_{p}$. Since every $\{x\} \subset X$ is open in $X_{p}$, then $\{x\} \cap A \neq \varnothing_{s}$; i.e., $x \in A$. Thus $X \subset A$. Since on the other hand $\bar{X}=X_{p}$, then the space $X_{p}$ has exactly two dense subsets; namely $X$ and $X_{p}$. Since they both are open, then $X_{p}$ is submaximal.

Theorem 5.3. Every maximally connected space is submaximal.

Proof. Let $A$ be a dense subset of $X$. Since $(X, \tau(A))$ is connected according to Theorem 2.4, then $\tau=\tau(A)$ and hence $A \in \tau$.

Example 5.4. A connected submaximal space need not be maximally connected. Let $X=\{a, b, c, d\}$. Consider the following two topologies on $X: \tau=\{\varnothing,\{a\},\{b\},\{a, b\}$, $\{a, b, c\},\{a, b, d\}, X\}$ and $\sigma=\tau \cup\{a, c\}$. Since none of the non-trivial open sets in the two topologies is closed, then $\tau$ and $\sigma$ are connected. Hence $(X, \tau)$ is not maximally connected. On the other hand $(X, \tau)$ is submaximal, since each its subset $S$ is dense iff $A$ and $B \in S$ and every set satisfying this condition is open. 
Theorem 5.5. A submaximal $S$-space is a door space.

Proof. Let $A$ be a non-closed subset of $X$. Since $X$ is S-space, then $A$ is dense. Since $X$ is submaximal, then $A$ is open. Thus each subset of $X$ is either closed or open and hence $X$ is a door space.

Corollary 5.6. Infinite space with the cofinite topology is not submaximal.

Theorem 5.7. Every door space is submaximal.

Proof. Let $A \subset X$ be dense. Since $X$ is a door space, then $A$ is either open or closed. If $A$ is closed, then $A=\bar{A}=X$ and hence $A$ is open.

Theorem 5.8. For a space $X$ the following are equivalent:

(1) $X$ is submaximal.

(9) $\mathcal{D}^{0}(X)=\mathcal{D}(X)$.

Theorem 5.9. For a space $X$ the following are equivalent:

(1) $X$ is submacimal and irreducible.

(2) $\tau=\mathcal{D}(X) \cup\{\varnothing\}$.

Proof. The space $X$ is irreducible iff $\tau \backslash \varnothing \subset \mathcal{D}(X)$ and submaximal iff $\mathcal{D}(x) \subset \tau$. Thus condition (1) is true iff $\tau \backslash \varnothing \subset \mathcal{D}(X) \subset \tau$. If $X$ is non-void, then $\varnothing \notin \mathcal{D}(X)$ and so (1) is equivalent to (2). The same is true if $X=\varnothing$.

In what follows, we see that the spaces, where each subset is a $\mathcal{B}$-set, are precisely the submaximal spaces.

Theorem 5.10. For a space $X$ the following are equivalent:

(1) $X$ is submaximal.

(10) Every subset of $X$ is a $\mathcal{B}$-set.

(11) Every dense subset of $X$ is a $\mathcal{B}$-set.

Proof. (1) $\Rightarrow(10)$ is clear, since a space is submaximal iff every subset is locally closed and since every locally closed set is a $\mathcal{B}$-set.

(10) $\Rightarrow(11)$ is trivial.

(11) $\Rightarrow$ (1) Let $A \subset X$ be dense. By (11) $A=U \cap B$, where $U$ is open and $B$ is semi-closed. Since $A \subset B$, then $B$ is dense. Thus $\operatorname{Int} B=\operatorname{Int} \bar{B}=\operatorname{Int} X=X$ and hence $B=X$. Thus $A=U$ is open and so $X$ is submaximal.

In the next theorem $\operatorname{Pcl} A$ and $\operatorname{Pint} A$ denote the preclosure and the preinterior of $A$.

Theorem 5.11. For a space $X$ the following are equivalent:

(1) $X$ is submaximal.

(12) $\bar{A}=\operatorname{Pcl} A$ for each $A \subset X$.

(13) $\operatorname{Int} A=\operatorname{Pint} A$ for each $A \subset X$. 
Proof. (5) $\Rightarrow(12)$ The set $\operatorname{Pcl} A$ is preclosed and by (5) closed. Since $A \subset \operatorname{Pcl} A \subset$ $\bar{A}$, then $\operatorname{Pcl} A=\bar{A}$.

$(12) \Rightarrow(13) \operatorname{By}(12) \overline{X \backslash A}=\operatorname{Pcl}(X \backslash A) \Leftrightarrow X \backslash \operatorname{Int} A=X \backslash \operatorname{Pint} A \Leftrightarrow \operatorname{Int} A=\operatorname{Pint} A$.

(13) $\Rightarrow(5)$ If $A$ is preopen, then by (13) $A=\operatorname{Pint} A=\operatorname{Int} A$.

Theorem 5.12. For the topological sum $X=\sum_{i \in I} X_{i}$, the following conditions are equivalent:

(1) $X$ is submaximal.

(2) $X_{i}$ is submaximal for each $i \in I$.

Proof. (1) $\Rightarrow$ (2) Theorem 1.1.

(2) $\Rightarrow(1)$ Let $A \subset X$ be dense. Then $A \cap X_{i}$ is dense in $X_{i}$, for each $i \in I$. By (2) $A \cap X_{i}$ is open in $X_{i}$ and hence in $X$. Thus $A=\cup_{i \in I}\left(A \cap X_{i}\right)$ is open in $X$, i.e., $X$ is submaximal.

Remark 5.13. In [7] it is proved (Corollary 4) that if $\Pi X_{\alpha}$ is submaximal, then each $X_{\alpha}$ is submaximal. Here we note that the reverse is not true even for a finite family of spaces. If $X$ is the Sierpinski space $(X=\{a, b\}, \tau=\{\varnothing,\{a\}, X\})$, then $X$ is submaximal but $Y=X \times X$ is not, since $\{(a, a),(b, b)\}$ is a dense non-open subset of $Y$.

\section{References}

[1] N. Bourbaki, General Topology, Addison-Wesley, Mass., 1966.

[2] N. Bourbaki, Éléments de mathématique, Algèbre commutative, Chap. 2., Hermann, Paris, 1961.

[3] D. Janković, I. Reilly and M. Vamanamurthy, "On strongly compact topological spaces," $Q$ \& $A$ in General Topology, 6 (1) (1988), 29-40.

[4] N. Levine, "Generalized closed sets in topolgy," Rend. Circ. Mat. Palermo, 19 (2) (1970), 89-96.

[5] N. Levine, "Semi-open sets and semi-continuity in topological spaces," Amer. Math. Monthly, 70 (1963), 36-41.

[6] N. Levine, "The superset topology," Amer. Math. Monthly, 75 (1968), 745-746.

[7] R. A. Mahmoud and D. A. Rose, "A note on spaces via dense sets," Tamkang J. Math., 24 (3) (1993), 333-339.

[8] A. S. Mashhour, M. E. Abd El-Monsef and S. N. El-Deeb, "On pre-continuous and weak precontinuous mappings," Proc. Math. Phys. Soc. Egypt, 53 (1982), 47-53.

[9] A. S. Mashhour, M. E. Abd El-Monsef, I. A. Hasanein and T. Noiri, "Strongly compact spaces," Delta J. Sci., 8 (1) (1984), 30-46.

[10] T. Nieminen, Lectures in general topology, unpublished, (in Finnish).

[11] O. Njåstad, "On some classes of nearly open sets," Pacific J. Math., 15 (1965), 961-970.

[12] P. L. Sharma, "A. class of spaces in which compact sets are finite," Canadian Math. Bull., 24 (1981), 373-375.

[13] J. Tong, "On decomposition of continuity in topological spaces," Acta Math. Hung., 54 (1989), $51-55$.

[14] S. Willard, General Topology, Addison-Wesley, 1970. 